\title{
Diagnosis and Management of Upper Gastrointestinal Bleeding
}

\author{
Erwin Biecker, Jörg Heller, Volker Schmitz, Frank Lammert, Tilman Sauerbruch
}

\author{
SUMMARY \\ Introduction: Upper gastrointestinal (GI) bleeding is defined \\ as bleeding proximal to ligament of Treitz. Its clinical \\ presentations are hematemesis, melena stool, or even \\ fresh bleeding per rectum. This paper reviews the \\ diagnosis and treatment of upper Gl bleeding.
}

Methods: Selective literature review.

Results: Common causes of upper Gl bleeding are peptic ulcer disease, bleeding from gastroesophageal varices, angiodysplasias, and Mallory-Weiss lesions. The most important diagnostic intervention is endoscopy, which allows therapeutic interventions if needed. Peptic ulcer disease is treated endoscopically with injection therapy and endoclips. Acute bleeding from oesophageal varices is treated by banding. Endoscopic treatment is accompanied by medical treatment with proton pump inhibitors for the treatment of peptic ulcer disease, and vasoactive drugs for the treatment of bleeding oesophageal varices.

Discussion: Modern endoscopy affords good localization of the bleeding site and successful treatment for most patients with upper Gl bleeding.

Dtsch Arztebl Int 2008; 105(5): 85-94

DOI: $10.3238 / a r z t e b l .2008 .0085$

Key words: gastrointestinal bleeding, melena stool, hematemesis, gastroesophageal varices, banding, endoscopy

Medizinische Klinik und Poliklinik I, Universitätsklinikum Bonn: Dr. med. Dr. phil. Biecker, PD Dr. med. Heller, PD Dr. med. Schmitz, Prof. Dr. med. Lammert, Prof. Dr. med. Sauerbruch

HELIOS Klinikum Siegburg, Klinik für Gastroenterologie/Hepatologie: Dr. med. Dr. phil. Biecker leeding of variable severity can occur anywhere in the gastrointestinal tract. Upper gastrointestinal (GI) bleeding is defined as bleeding proximal to the ligament of Treitz.

\section{General observations}

The incidence of upper gastrointestinal bleeding is approximately 50 per 100000 persons per year (1). The mortality of this condition is between $5 \%$ and $11 \%$ (2). The main sources of bleeding are peptic ulcers, esophagitis, drug-induced mucosal damage, sequelae of portal hypertension (esophageal varices, varices of the gastric fundus, portal hypertensive gastropathy), vascular anomalies, traumatic and postoperative lesions, and tumors (see box 1). The classic clinical signs are the regurgitation or vomiting of blood (hematemesis) and black, tarry stool (melena). Severe, brisk bleeding can also present with the passage of bright red blood per rectum (hematochezia). Insidious upper gastrointestinal bleeding can present with non-specific signs such as fatigue, prostration, shortness of breath, or angina pectoris.

Occasionally, the only finding pointing to a gastrointestinal hemorrhage is a laboratory result, such as iron-deficiency anemia or a positive test for occult blood in the stool. The stool can be tarry if 50 to $100 \mathrm{~mL}$ of blood are lost per day. Hemodynamically relevant manifestations, e.g., hypotension in a supine patient, arise after loss of $20 \%$ to $25 \%$ of the total intravascular blood volume and indicate the presence of massive hemorrhage. Patients with orthostatic tachycardia and hypotension have lost at least $10 \%$ to $20 \%$ of their intravascular blood volume. Alongside these hemodynamic parameters indicating the severity of the hemorrhage, a number of clinical signs provide clues to its localization as well as to possible accompanying illnesses:

- Tarry stool and hematemesis indicate upper gastrointestinal bleeding.

\section{The classic signs of gastrointestinal bleeding are \\ - Regurgitation or vomiting of blood (hematemesis) \\ - Tarry stool}




\section{BOX 1}

\section{Causes of upper gastrointestinal bleeding*1}

- Peptic ulcer

- Esophagitis

- Drug-induced mucosal damage (NSAID)

- Ulcer

- Erosions

- Traumatic or postoperative lesions

- Mallory-Weiss lesion

- Arterio-intestinal fistula

- Malignant tumor

- Sequelae of portal hypertension

- Esophageal varices

- Varices of the gastric fundus

- Portal hypertensive gastropathy

- Vascular anomalies

- Dieulafoy lesion

- Gastric antral vascular ectasia (GAVE syndrome)

- Angiodysplasia

- Rendu-Osler-Weber syndrome (hereditary hemorrhagic telangiectasia)

- Bleeding from the hepato-pancreatico-biliary system

- Bleeding from a duodenal diverticulum

${ }^{\star 1}$ modified from (25)

\section{TABLE}

The risk of recurrent bleeding from an ulcer after exclusively medical therapy, depending on the Forrest classification of the ulcer*1

\begin{tabular}{l|c|c}
$\begin{array}{l}\text { Forrest classification: } \\
\text { endoscopic appearance }\end{array}$ & $\begin{array}{c}\text { Frequency } \\
(\%)\end{array}$ & $\begin{array}{c}\text { Risk of recurrent } \\
\text { bleeding within 24 hours } \\
\text { after medical } \\
\text { therapy (\%) }\end{array}$ \\
\hline la: Active arterial hemorrhage & 10 & 90 \\
\hline Ila: Visible vessel that is not bleeding & 25 & 50 \\
\hline Ilb: Adherent clot & 10 & $25-30$ \\
\hline Ib: Oozing hemorrhage without visible source & 10 & $10-20$ \\
\hline Ilc: Hematin & 10 & $7-10$ \\
\hline III: Normal base of the ulcer & 35 & $3-5$ \\
\hline
\end{tabular}

${ }^{* 1}$ modified from (26)
- Hematochezia indicates lower gastrointestinal bleeding or massive bleeding distal to the pylorus of the stomach.

- Ascites and jaundice indicate hepatic failure and imply that the source of bleeding may be dilated venous collaterals in a patient with hepatic cirrhosis.

The following points in the history require attention:

- Has the patient regularly been taking any medications that can provoke hemorrhage, such as nonsteroidal anti-inflammatory drugs (NSAID), anticoagulants, or platelet aggregation inhibitors?

- Has the patient ever had a similar episode before (variceal hemorrhage, diverticular hemorrhage)?

- Have any prostheses been inserted that might bear a causal relationship to the hemorrhage (coronary stents and platelet aggregation inhibitors, cardiac valvular prostheses and anticoagulants, aortic prostheses with the risk of an aorto-intestinal fistula)?

If endoscopy cannot be performed immediately, two bedside tests may be useful, both of which are relatively specific for upper intestinal bleeding, though not very sensitive:

1. rectal examination with examination of the stool for its color and consistency (melena);

2. the insertion of a nasogastric tube to diagnose or exclude severe upper gastrointestinal hemorrhage.

The initial laboratory tests include a blood count, INR, PTT, electrolytes, creatinine, and blood typing and cross-matching in case transfusion should be necessary. Every patient with a severe intestinal hemorrhage, particularly if esophageal varices are suspected or the hemorrhage is already hemodynamically significant, should first be hemodynamically stabilized in an intensive care or step-down unit. Further diagnostic measures should be undertaken once the patient is hemodynamically stable.

\section{Clinical assessment and risk estimation}

The most important initial assessment is the measurement of the patient's pulse and blood pressure. The physical examination can also provide clues to the source of the hemorrhage. The main risk factors that are evident on clinical examination are hemodynamic instability, a drop of the hematocrit by more than $6 \%$, and any evidence of active bleeding (hematemesis, hematochezia).

\section{Important points in the history}

- Medications that promote bleeding?

- Any earlier episodes of hemorrhage?

- Are there any implanted prostheses that might be causally related to the hemorrhage?

\section{Initial treatment}

Every patient with a severe gastrointestinal hemorrhage must first be hemodynamically stabilized. 
A number of classification systems have been proposed for assessing the patient's prognosis. Unfavorable prognostic factors include the following:

- Age over 65 years

- More than two concomitant illnesses

- An unfavorable site of bleeding (posterior wall of the duodenal bulb)

- Clinical signs of severe bleeding (hematemesis, hematochezia)

- Hemodynamic instability

- Active bleeding during endoscopy.

A number of scoring systems have been developed to assess the prognosis after bleeding from peptic ulcer disease (Baylor Score, Rockall Score, Cedars-Sinai Index). It has not yet been conclusively determined which of these scoring systems is best in clinical practice (3).

\section{Diagnostic assessment and the causes of hemorrhage}

The most important diagnostic step is upper gastrointestinal endoscopy. In cases of hemodynamically relevant hemorrhage, endoscopy should be performed as soon as the patient has been clinically stabilized (although no controlled studies on the ideal timing of endoscopy are yet available). A standard endoscope is used, or, if the hemorrhage is massive, an endoscope with a wide working tube. If a rare source of hemorrhage is suspected, such as bleeding from the papilla, the device can be switched to one with side-view optics. The source of bleeding can be localized by endoscopy in about $90 \%$ of patients with upper gastrointestinal hemorrhage (2).

\section{Sources of bleeding and treatment Bleeding ulcers}

Bleeding from ulcers is the most common type of upper GI bleeding (30\% to $40 \%$, though the percentage estimates vary widely). About $60 \%$ of bleeding ulcers are located in the duodenum (figure 1), while $40 \%$ are in the stomach. Bleeding ulcers are classified by the Forrest criteria (4) (box 3); this classification provides a means of estimating the risk of continued or recurrent bleeding if purely supportive treatment is provided, without local hemostasis (table). All patients with active bleeding or with endoscopic signs of bleeding, such as a vascular stump or an adherent blood clot, should be treated endoscopically, e.g., by the injection of suprarenin solu-

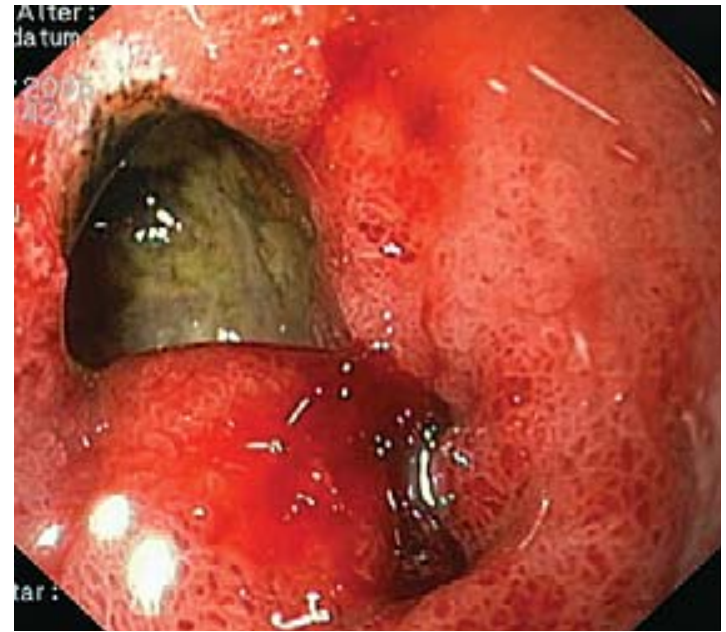

Figure 1:

Endoscopic photograph obtained in a 72 year-old woman with a severe oozing hemorrhage from a duodenal ulcer (Forrest lb hemorrhage). The ulcer extends to the pylorus. tion (1:10 000), local argon plasma coagulation (APC), or clipping of the bleeding vessel with one or more endoclips. Adherent clots should be irrigated away; if a clot cannot be removed by irrigation, one can first inject suprarenin into the underlying vessel (to prevent hemorrhage), then remove the clot with a polypectomy loop and, finally, treat the lesion beneath the clot endoscopically (5). Initial hemostasis is successful in $70 \%$ of patients, including high-risk patients (Forrest Ia hemorrhage from an ulcer on the posterior duodenal wall).

Multiple meta-analyses of clinical trials have confirmed that endoscopic treatment significantly improves patients' prognosis with respect to recurrent hemorrhage and the need for transfusions and surgery (level 1a evidence, grade A recommendation). An older metaanalysis (6) already showed that local endoscopic therapy raises the probability of not sustaining a recurrent hemorrhage, or of not requiring surgery, by $60 \%$.

It is still unclear what the best form of local treatment is among the various ones that are currently available. Combination therapy is probably the most effective approach, e.g., suprarenin injection followed by clipping (7; level $1 \mathrm{~b}$ evidence, grade A recommendation [see also box 2]).

Controlled studies have shown that the administration of proton pump inhibitors for 72 hours right after endoscopic intervention significantly lowers the risk of recurrent hemorrhage (8). Omeprazole is the beststudied proton pump inhibitor; $80 \mathrm{mg}$ are given initially, followed by a continuous infusion of $8 \mathrm{mg} /$ hour. The

\section{Poor prognostic signs}

- Age > 65 years

- More than two types of concomitant illness

- Hemodynamic instability

- Brisk, active bleeding during endoscopy

\section{Diagnostic assessment}

The most important method is endoscopy of the upper gastrointestinal tract with a standard endoscope, or, in the case of active bleeding, an endoscope with a wide working tube. 
BOX 2

\section{The evidence levels used in treatment recommendations ${ }^{* 1}$}

1a - Meta-analyses of randomized, controlled studies

1b - At least one randomized, controlled study with a narrow confidence interval

1c - So-called "all-or-none" studies, i.e., the new treatment is found to be markedly better than the previous one

$2 a$ - Meta-analyses of cohort studies

$2 b$ - A small number of cohort studies or randomized, controlled studies of low quality

2c - Outcome studies

3a - Meta-analyses of case-control studies

3b - A small number of case-control studies

4 - Case series, cohort studies, and case-control studies of low quality

5 - Expert opinion or purely experimental findings

The "strength" or weighting of a recommendation depends on the level of the evidence on which it rests:

Grade A - Requires level 1 evidence

Grade B - Requires level 2 or 3 evidence or extrapolations from level 1 trials

Grade C - Requires level 4 evidence or extrapolations from level 2 or 3 trials

Grade D - Requires level 5 evidence or conflicting evidence from trials of any other level

\footnotetext{
${ }^{* 1}$ For the purposes of this article, we have adopted the classification scheme for evidence and recommendations used by the 0xford Centre for Evidence Based Medicine: see www.cebm.net/ levels_of_evidence.asp
}

\section{BOX 3}

\section{The Forrest classification of ulcer hemorrhage (4)}

- la: Active arterial hemorrhage

- Ib: Oozing hemorrhage

- lla: Visible vascular stump that is currently not bleeding

- Ilb: Adherent clot

- Ilc: Hematin in the base of the ulcer

- III: No visible source of bleeding

Cochrane analyses on the subject were published in $2006(12,13)$. In the analysis by Leontiadis et al., only high-dose PPI administration was found to lower the rate of surgical intervention significantly. High-dose therapy was defined, in this context, as the administration of more than $120 \mathrm{mg}$ over 24 hours, e.g., as an $80 \mathrm{mg}$ bolus followed by a continuous infusion of $8 \mathrm{mg}$ per hour (level 1a evidence, grade A recommendation) (13). Neither of these two meta-analyses showed a reduction in mortality $(12,13)$. It must be pointed out, however, that some of the studies included in these analyses were performed in Asian countries. It is known that persons of Asian ethnic origin metabolize PPI in a different way than persons of European origin; thus, the study results are not necessarily generalizable to patients in Europe.

Though these data are encouraging, there remains controversy as to whether proton pump inhibitors ought to be given intravenously after successful hemostasis by endoscopic therapy. A prospective international study is currently being performed to answer this question; its results have not yet been announced.

In the authors' institution, patients with bleeding ulcers are initially given $40 \mathrm{mg}$ of omeprazole twice daily, or an equivalent amount of another proton pump inhibitor, intravenously for at least three days. The dose is adjusted thereafter depending on the size of the ulcer and the further course of healing, until the route of administration is finally switched from intravenous to oral.

After initially successful treatment, it is important to lower the risk of recurrent hemorrhage permanently. The successful eradication of an associated Helicobacter pylori infection lowers the risk of reappearance of an ulcer in the first year after hemorrhage to less than $5 \%$ (14) (level $1 \mathrm{~b}$ evidence, grade $\mathrm{A}$ recommendation).

\section{Sources of hemorrhage}

Ulcers are the most common source of upper gastrointestinal hemorrhage (35\%).

\section{Treatment}

Local endoscopic therapy raises the probability of not suffering any further hemorrhages, and of not needing surgery, by $60 \%$. 
Therefore, every patient should already be tested for Helicobacter pylori at the initial endoscopy. The sensitivity of the rapid Helicobacter pylori test has not yet been affected when the initial endoscopy is performed, but then drops considerably once PPI treatment is begun. At the same time, the possibility of a drug-induced ulcer must be definitively confirmed or ruled out. If nonsteroidal anti-inflammatory drugs (NSAID) have played a contributory role in the hemorrhage, they should either be discontinued or - if they are absolutely necessary - continued in combination with a PPI. It is not yet known for certain whether it is better to give a COX-2-selective NSAID together with a PPI than to give a non-selective COX inhibitor together with a PPI. In any case, this combination was used successfully in high-risk patients in a recent study (15) with a median follow-up interval of 13 months. It is important to point out that this study was performed in Hong Kong, and its results are not necessarily generalizable to Germany. The patients in the study population were of Asian descent and therefore metabolized medications differently from European patients; thus, the same study, if performed in Europe, might have had a different result. Other pharmacological therapies aimed at stopping hemorrhage, e.g., the administration of tranexamic acid, somatostatin, and analogues of these substances, have been found to have only a small effect, if any, and are not currently a part of standard care.

In a few studies, the use of a motilin agonist (erythromycin $250 \mathrm{mg} \mathrm{IV}$ ) before endoscopy has been found to improve the conditions for emergency endoscopy to a significant degree, because it speeds the passage of blood and clot through the stomach (16) (level $2 \mathrm{~b}$ evidence, grade $\mathrm{B}$ recommendation).

It is difficult to say under what circumstances surgery is indicated, because there are no prospective, controlled studies to provide a reliable answer to this question (17). Surgery is certainly indicated, however, when any of the following is the case:

- when the patient cannot be hemodynamically stabilized despite adequate volume replacement and medical therapy,

- when recurrent hemorrhage occurs despite initial stabilization by endoscopic treatment and reendoscopy, or

- when there is persistent hemorrhage requiring the transfusion of more than three units of erythrocyte concentrate per day.

\section{Risk reduction}

High-dose PPI therapy significantly lowers the rate of surgical intervention.

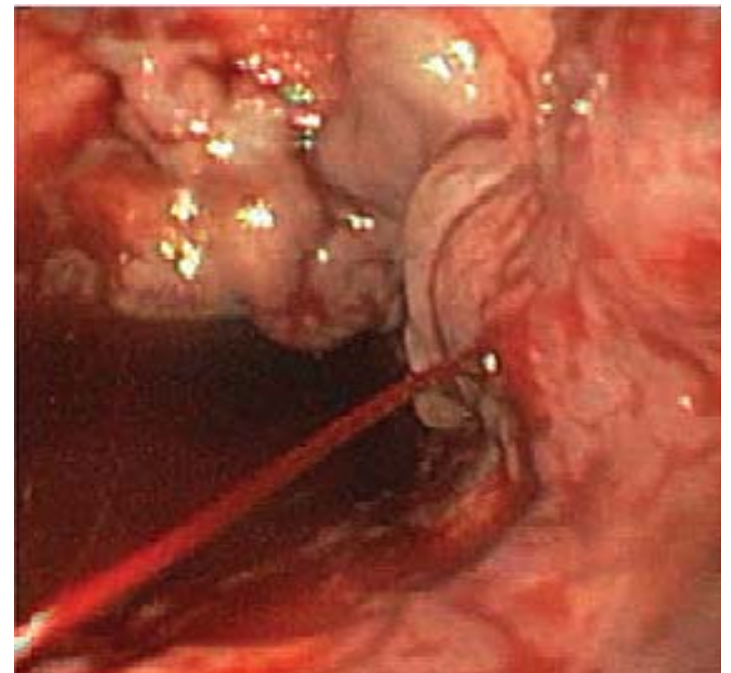

Figure 2: Active (spurting) hemorrhage from an esophageal varix in a 53-year-old man with alcoholic cirrhosis of the liver.

Surgical consultation should always be obtained for patients with severe, active hemorrhage.

Interventional radiological techniques can be an alternative to surgery when the necessary apparatus and trained personnel are at hand. Selective catheterization and angiography can be used to localize the bleeding vessel, which can then be embolized, e.g., with metallic coils (18). However, $10 \%$ to $20 \%$ of patients treated in this way will have a recurrent hemorrhage within 72 hours.

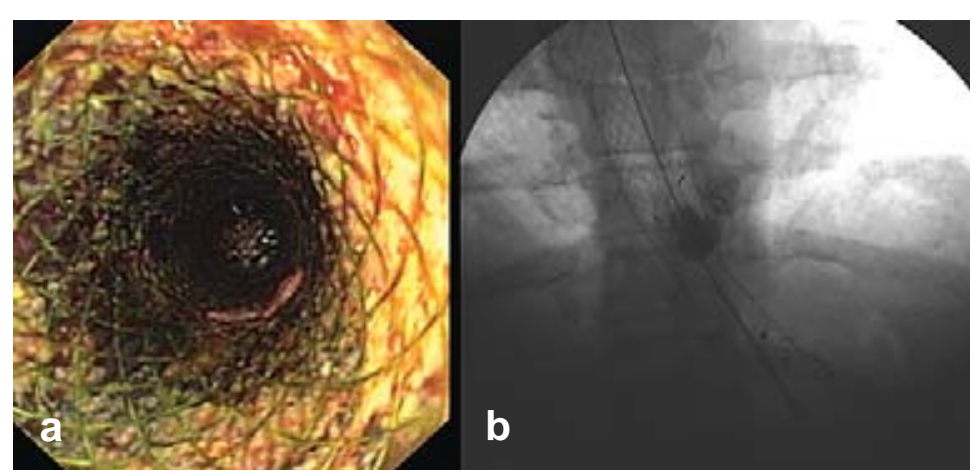

Figure 3: a) Endoscopic and b) radiological images obtained in a 41-year-old woman with alcoholic cirrhosis of the liver and recurrent, severe variceal hemorrhages, treated in the past with Histoacryl (n-butyl-2-cyanoacrylate) and other methods. The bleeding could only be stopped with the placement of a compression stent.

\section{The risk of recurrence}

The successful eradication of an associated Helicobacter pylori infection reduces the risk of appearance of a new ulcer in the first year after an ulcer-related hemorrhage to less than $5 \%$. 
Hemorrhage from collateral vessels in portal hypertension

Hemorrhage from collateral vessels, usually esophageal varices, in the setting of portal hypertension is one of the more common causes of upper gastrointestinal hemorrhage (figure 2) (19). The prognosis is poor. Up to $30 \%$ of these patients, most of whom have hepatic cirrhosis, die as a result of the initial bleed (20). As soon as the clinical suspicion of a variceal hemorrhage arises, vasoactive treatment should be initiated, e.g., with $1.5 \mathrm{mg}$ of terlipressin IV every 4 to 6 hours in a $70-\mathrm{kg}$ patient (level 1a evidence, grade A recommendation), unless contraindicated by severe coronary heart disease or other comorbidities. This treatment is continued for 2 to 5 days (21). The same holds for antibiotic prophylaxis, e.g., with ciprofloxacin $500 \mathrm{mg}$ po bid (22). The administration of antibiotics lowers the recurrence rate (23) (level $1 \mathrm{~b}$ evidence, grade A recommendation). In one study, only 4 of 59 patients treated with antibiotics had a recurrent hemorrhage within 7 days of the initial bleed, as compared with 21 of 61 patients not treated with antibiotics (23).

Endoscopy should be performed as rapidly as possible in all patients in whom variceal bleeding is suspected. If active variceal bleeding is found, or if endoscopy reveals varices and blood in the stomach without any other evident source of hemorrhage, then endoscopic therapy should be provided immediately. This usually involves ligation of the varices.

Because varices of the gastric fundus cannot be treated safely by ligation, Histoacryl (n-butyl-2-

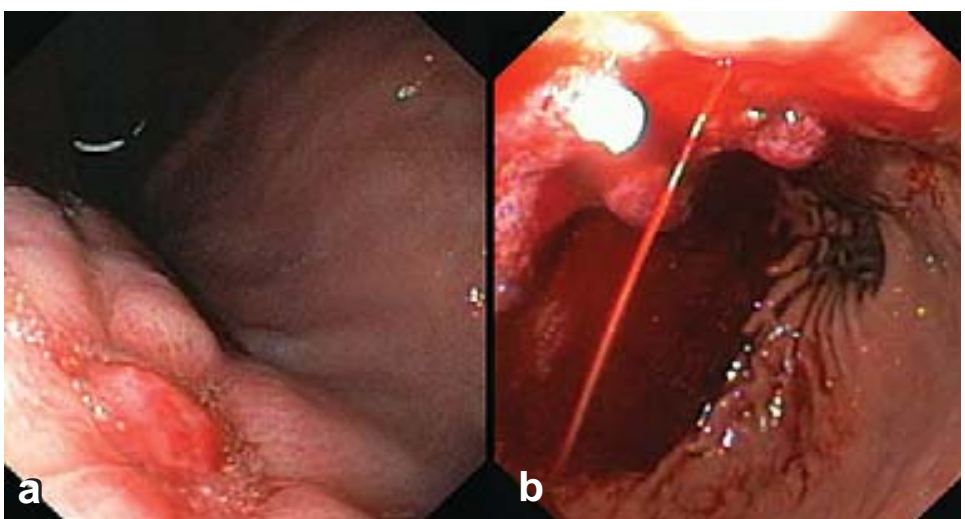

Figure 4: A Dieulafoy ulcer at the corpus-antrum junction. a) The lesion was seen in an inverted state in the initial endoscopy. b) A spurting hemorrhage (Forrest la) was seen after the endoscopic device touched the lesion. This 22-year-old woman had come to the emergency room because of massive hematemesis.

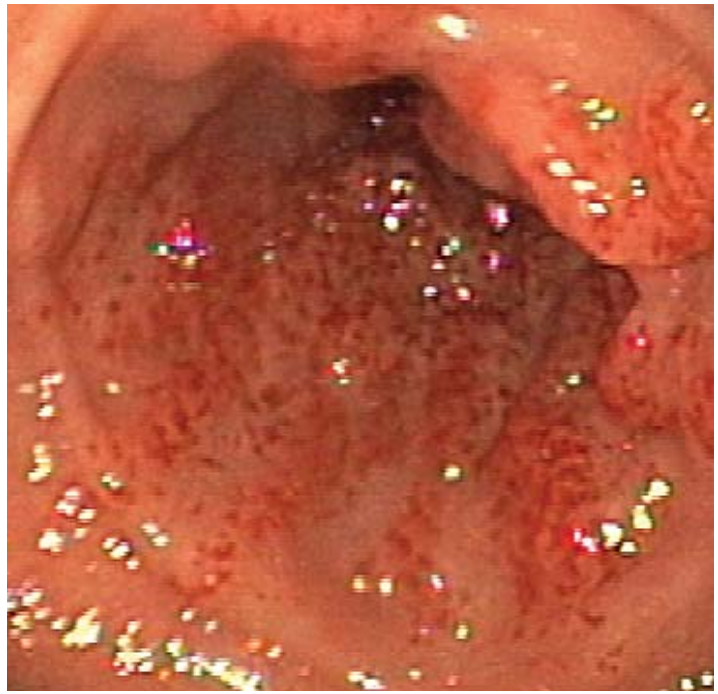

Figure 5: Typical vascular changes in the antrum of the stomach of a 63-year-old woman with chronic blood-loss anemia due to gastric antral vascular ectasia (the GAVE syndrome).

cyanoacrylate) is injected into them instead (level $1 \mathrm{~b}$ evidence, grade A recommendation).

If active variceal bleeding cannot be stopped endoscopically, local balloon tamponade must be performed as a temporizing measure, for no longer than 24 hours (level 4 evidence, grade $\mathrm{C}$ recommendation). Very recently, soft endoluminal stents have also been used to compress varices (figure 3). Only limited experience has been obtained with this technique to date.

Patients whose acute variceal bleeding persists despite endoscopic therapy can be treated by the insertion of a transjugular intrahepatic porto-systemic stent shunt (TIPS) (level $2 \mathrm{~b}$ evidence, grade $\mathrm{B}$ recommendation). In the authors' institution, the placement of an "emergency TIPS" has obviated the need for "emergency shunt surgery" in most patients. Nonetheless, this procedure does not reduce mortality to any significant degree for patients with severely decompensated hepatic cirrhosis, even if the bleeding can be successfully stopped.

\section{Mallory-Weiss tears}

Deep, bleeding tears of the mucosa at the gastroesophageal junction are found in 5\% to $10 \%$ of all patients

\section{Indications for surgery}

- Hemodynamic instability despite adequate volume resuscitation

- Recurrent hemorrhage after two endoscopic hemostatic procedures

- Persistent bleeding requiring the transfusion of more than three units of red cells per day

\section{Bleeding from esophageal varices}

Up to $30 \%$ of patients die from their initial hemorrhage. 


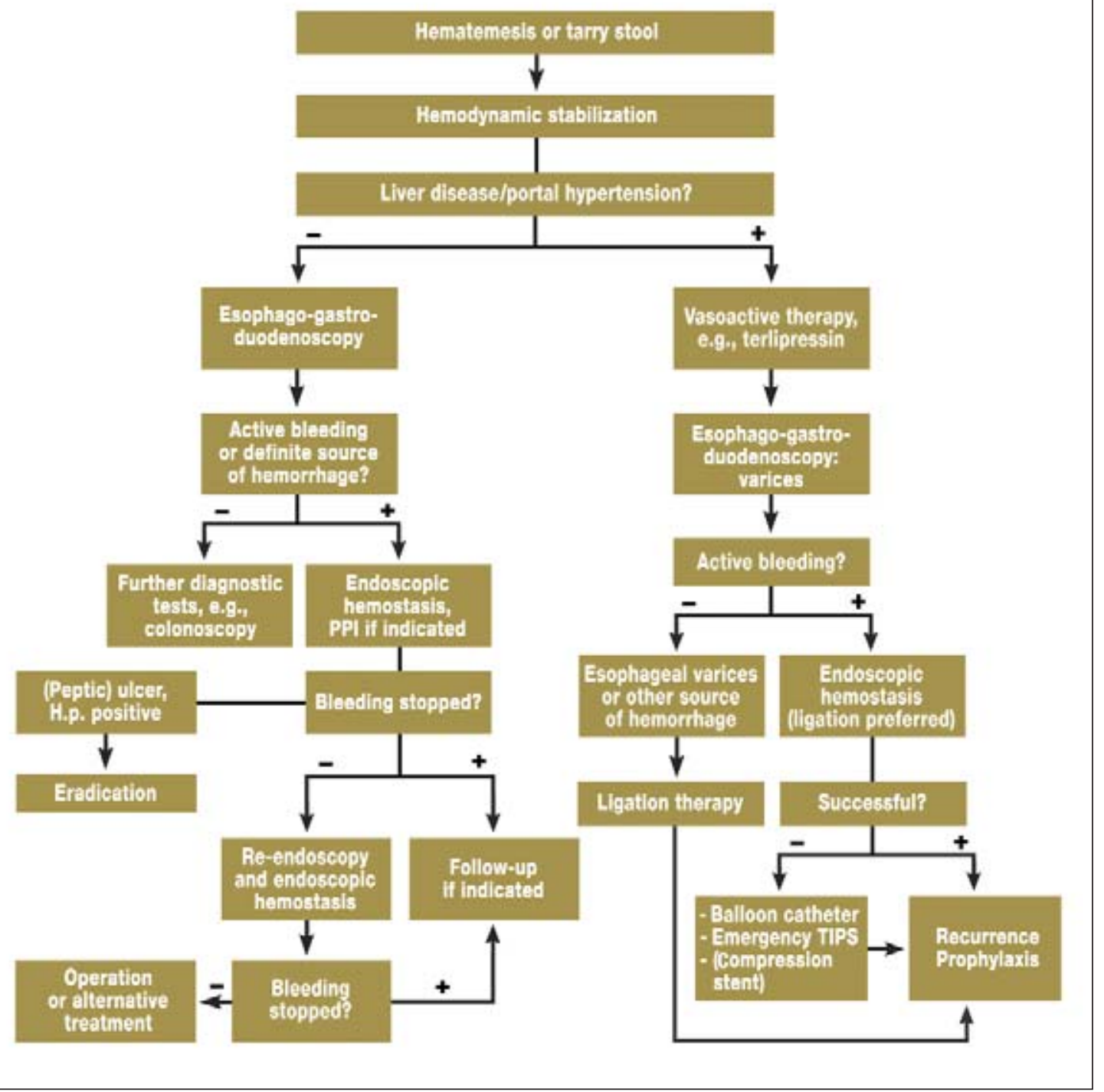

The diagnostic and therapeutic flowchart for upper gastrointestinal bleeding that was developed in the authors' clinic. TIPS, transjugular intrahepatic portalsystemic stent shunt; PPI, proton pump inhibitor; H.p., Helicobacter pylori

with upper gastrointestinal bleeding. Usually, tearing of this type is precipitated by vomiting or coughing fits. Alcoholic persons are predisposed to Mallory-Weiss tears. Often, the bleeding stops spontaneously. If the lesion is actively bleeding, hemostasis should be obtained via endoscopy, either with the application of clips or with rubber-band ligation (24) (level 2b evidence, grade B recommendation).

\section{Hemorrhagic-erosive changes}

Hemorrhagic-erosive changes can occur in the esophagus and in the stomach. Such changes in the esophagus are often due to reflux esophagitis or to medications; in the stomach, they are often associated with non-steroidal anti-inflammatory drugs (NSAID), alcoholism, or stress lesions in patients receiving intensive care. Stress-induced gastric lesions most

\section{Varices of the gastric fundus}

Varices of the gastric fundus cannot be safely treated with ligation and are, therefore, treated by the injection of tissue sealants.

\section{TIPS}

The insertion of a transjugular intrahepatic portal-systemic stent shunt (TIPS) is a therapeutic option when the endoscopic treatment of an acute variceal hemorrhage is unsuccessful. 
commonly occur in patients with burns or neurological illnesses. Local hemostasis is only rarely needed in such cases. The most important form of treatment here consists of recurrence prophylaxis with proton pump inhibitors.

\section{Rarer causes of upper gastrointestinal hemorrhage}

If endoscopy initially fails to reveal any source of hemorrhage even though an upper gastrointestinal bleed has unquestionably occurred, as manifested by the finding of blood or blood clots in the lumen of the gastrointestinal tract, then rarer causes of hemorrhage should be considered in the differential diagnosis (box 1). Hemorrhages from large, aberrant vascular ectasias with small overlying mucosal defects, usually in the gastric fundus (Dieulafoy lesion, illustrated in figure 4) are particularly dangerous. These have been treated successfully with injection therapy and endoclip application as well as with rubber-band ligation (level $3 \mathrm{~b}$ evidence, grade $\mathrm{C}$ recommendation).

Rarely, gastric antral vascular ectasia (the GAVE syndrome, also called "watermelon stomach") can be the cause of a gastrointestinal bleed (figure 5). GAVE syndrome often arises in connection with portal hypertension. Repeated argon gas plasma coagulation is a treatment option with a good chance of success (level 4 evidence, grade $\mathrm{C}$ recommendation).

Another rare cause of upper gastrointestinal hemorrhage is bleeding from the hepato-bilio-pancreatic system (at endoscopy, blood is seen to be coming out of the papilla). Duodenal diverticula, if present, can also be a site of hemorrhage. Like colonic diverticula, these are treated with injection therapy and/or endoclips.

Vasculitis should be considered in the differential diagnosis if the patient has cutaneous changes and the autoantibodies that are typical of this entity. Vasculitis of the intermediate-sized and small vessels, as is seen in Churg-Strauss syndrome or polyarteritis nodosa, can cause ischemic ulceration and, therefore, hemorrhage. The treatment is directed at the underlying illness; endoscopic intervention is rarely needed to control the bleeding.

Aorto-enteric fistulae usually arise after surgery for an aortic aneurysm and are a source of special danger. This possibility is usually suggested by the patient's history or by the findings of computed tomography. There is usually no way to treat a bleeding aorto-enteric fistula endoscopically and the patient must therefore be taken to surgery immediately.
The authors have developed a diagnostic and therapeutic algorithm for upper GI bleeding that is currently in use in their institution (figure 6).

\section{Conclusion}

Gastrointestinal bleeding is frequently encountered in clinical practice and becomes more common with advancing age. It is vitally important to recognize the patients that are in acute danger from hemorrhage as soon as they present to medical attention. These patients should be hemodynamically stabilized and should then undergo endoscopy as soon as possible thereafter. If the source of the bleeding is found, it can usually be directly treated with endoscopic therapy.

\section{Conflict of interest statement}

Dr. Heller has received lecture honoraria from Meduna Arzneimittel GmbH. Dr. Lammert has received lecture honoraria from the Falk Foundation. Prof. Sauerbruch has received lecture honoraria from the Falk Foundation and third-party funds from various companies for continuing medical education events at the University of Bonn Medical Department. Dr. Biecker and Dr.

Schmitz state that they have no conflict of interest as defined by the guidelines of the International Committee of Medical Journal Editors.

Manuscript received on 26 June 2007; revised version accepted on 17 December 2007.

Translated from the original German by Ethan Taub, M.D.

\section{REFERENCES}

1. Rollhauser C, Fleischer DE: Nonvariceal upper gastrointestinal bleeding. Endoscopy 2004; 36: 52-8.

2. Ell C, Hagenmuller F, Schmitt W, Riemann JF, Hahn EG, Hohenberger W: Multizentrische prospektive Untersuchungen zum aktuellen Stand der Therapie der Ulkusblutung in Deutschland. Dtsch Med Wochenschr 1995; 120: 3-9.

3. Camellini L, Merighi A, Pagnini C et al.: Comparison of three different risk scoring systems in non-variceal upper gastrointestinal bleeding. Dig Liver Dis 2004; 36: 271-7.

4. Forrest JA, Finlayson ND, Shearman DJ: Endoscopy in gastrointestinal bleeding. Lancet 1974; 2: 394-7.

5. Kahi CJ, Jensen DM, Sung JJ et al.: Endoscopic therapy versus medical therapy for bleeding peptic ulcer with adherent clot: a metaanalysis. Gastroenterology 2005;129: 855-62.

6. Cook DJ, Guyatt GH, Salena BJ, Laine LA: Endoscopic therapy for acute non-variceal upper gastrointestinal hemorrhage: a metaanalysis. Gastroenterology 1992;102: 139-48

7. Goto H, Ohta S, Yamaguchi Y, Yukioka T, Matsuda H, Shimazaki S Prospective evaluation of hemoclip application with injection of epinephrine in hypertonic saline solution for hemostasis in unstable patients with shock caused by upper Gl bleeding. Gastrointest Endosc 2002; 56: 78-82.

8. Lau JY, Sung JJ, Lee KK et al.: Effect of intravenous omeprazole on recurrent bleeding after endoscopic treatment of bleeding peptic ulcers. N Engl J Med 2000; 343: 310-6.

\section{Rarer causes of upper gastrointestinal bleeding \\ - Bleeding from large, aberrant vascular ectasias \\ - Aorto-intestinal fistula \\ - Bleeding from the hepato-bilio-pancreatic system}


9. Udd M, Miettinen P, Palmu A et al.: Regular-dose versus high-dose omeprazole in peptic ulcer bleeding: a prospective randomized double-blind study. Scand J Gastroenterol 2001; 36: 1332-8.

10. Javid G, Masoodi I, Zargar SA et al.: Omeprazole as adjuvant therapy to endoscopic combination injection sclerotherapy for treating bleeding peptic ulcer. Am J Med 2001; 111: 280-4.

11. Lau JY, Leung WK, Wu JC et al.: Omeprazole before endoscopy in patients with gastrointestinal bleeding. N Engl J Med 2007; 356: 1631-40.

12. Dorward S, Sreedharan A, Leontiadis GI, Howden CW, Moayyedi P, Forman D: Proton pump inhibitor treatment initiated prior to endoscopic diagnosis in upper gastrointestinal bleeding. Cochrane Database Syst Rev 2006(4): CD005415.

13. Leontiadis Gl, Sharma VK, Howden CW: Proton pump inhibitor treatment for acute peptic ulcer bleeding. Cochrane Database Syst Rev 2006(1): CD002094.

14. Sung JJ, Chung SC, Ling TK et al.: Antibacterial treatment of gastric ulcers associated with Helicobacter pylori. N Engl J Med 1995; 332 139-42.

15. Chan FK, Wong VW, Suen BY et al.: Combination of a cyclooxygenase-2 inhibitor and a proton-pump inhibitor for prevention of recurrent ulcer bleeding in patients at very high risk: a doubleblind, randomised trial. Lancet 2007; 369: 1621-6.

16. Carbonell N, Pauwels A, Serfaty L, Boelle PY, Becquemont L, Poupon R: Erythromycin infusion prior to endoscopy for acute upper gastrointestinal bleeding: a randomized, controlled, doubleblind trial. Am J Gastroenterol 2006; 101: 1211-5.

17. Imhof M, Ohmann C, Roher HD, Glutig H: Endoscopic versus operative treatment in high-risk ulcer bleeding patients - results of a randomised study. Langenbecks Arch Surg 2003; 387: 327-36.

18. Miller MJ, Smith T: Angiographic diagnosis and endovascular management of nonvariceal gastrointestinal hemorrhage. Gastroenterol Clin North Am 2005; 34: 735-52.

19. Biecker E, Heller J, Appenrodt B, Schepke M, Sauerbruch T: Gastrointestinal bleeding in portal hypertension in liver cirrhosis. Z Gastroenterol 2005; 43: 35-46.

20. Schepke M, Kleber G, Nurnberg D et al.: Ligation versus propranolol for the primary prophylaxis of variceal bleeding in cirrhosis. Hepatology 2004; 40: 65-72.

21. de Franchis R: Evolving consensus in portal hypertension. Report of the Baveno IV consensus workshop on methodology of diagnosis and therapy in portal hypertension. J Hepatol 2005; 43: 167-76.

22. Appenrodt B, Sauerbruch T: Antibiotic therapy and prophylaxis in liver cirrhosis and infection. Dtsch Med Wochenschr 2007; 132: 623-6.

23. Hou MC, Lin HC, Liu TT et al.: Antibiotic prophylaxis after endoscopic therapy prevents rebleeding in acute variceal hemorrhage: a randomized trial. Hepatology 2004; 39: 746-53.

24. Chung IK, Kim EJ, Hwang KY et al.: Evaluation of endoscopic hemostasis in upper gastrointestinal bleeding related to MalloryWeiss-Syndrome. Endoscopy 2002; 34: 474-9.
25. Rockall TA, Logan RF, Devlin HB, Northfield TC: Incidence of and mortality from acute upper gastrointestinal haemorrhage in the United Kingdom. Steering Committee and members of the National Audit of Acute Upper Gastrointestinal Haemorrhage. BMJ 1995; 311: 222-6.

26. Katschinski B, Logan R, Davies J, Faulkner G, Pearson J, Langman M: Prognostic factors in upper gastrointestinal bleeding. Dig Dis Sci 1994; 39: 706-12.

\section{Correpsonding author}

Prof. Dr. med. Tilman Sauerbruch

Direktor der Medizinischen Klinik und Poliklinik I

Universitätsklinikum Bonn

Sigmund-Freud-Str. 25

53105 Bonn, Germany

sauerbruch@uni-bonn.de

\section{Further Information}

This article has been certified by the North Rhine Academy for Postgraduate and Continuing Medical Education. Deutsches Ärzteblatt provides certified continuing medical education (CME) in accordance with the requirements of the Chambers of Physicians of the German federal states (Länder). CME points of the Chambers of Physicians can be acquired only through the Internet by the use of the German version of the CME questionnaire within 6 weeks of publication of the article. See the following website: www.aerzteblatt.de/cme

Participants in the CME program can manage their CME points with their 15-digit "uniform CME number" (einheitliche Fortbildungsnummer, EFN). The EFN must be entered in the appropriate field in the www.aerzteblatt.de website under "meine Daten" ("my data"), or upon registration. The EFN appears on each participant's CME certificate.

The solutions to the following questions will be published in Volume 12/2008. The CME unit "The Treatment of Deep Vein Thrombosis in the Pelvis and Leg" (Volume 1-2/2008) can be accessed until the 18th February. For volume 9/2008 we plan to offer the topic "Basic knowledge of Refractive Surgery"

Solutions to the CME questionnaire in Volume 49/2007: Löser C: Involuntary Weight Loss in Elderly People: 1/e, 2/b, 3/c, 4/b, 5/b, 6/c, 7/c, 8/d, 9/e, 10/b 


\section{Question 1}

Which of the following simple diagnostic measures may yield clues to the source of a gastrointestinal hemorrhage?

a) Examination of the pupils and carbon-14 breathing test

b) Esophageal manometry and test for occult blood in the stool

c) Insertion of a nasogastric tube and rectal stool examination

d) Venous and arterial blood gas analysis

e) Blood count and ECG

Question 2

What is the most common cause of upper

gastrointestinal bleeding?
a) Duodenal ulcer
b) Gastric ulcer
c) Esophageal ulcer
d) Reflux esophagitis
e) Esophageal varices

\section{Question 3}

Which of the following clinical signs is seen in upper gastrointestinal bleeding?
a) Hematemesis
b) Fall of body temperature
c) Hemosiderosis
d) Bradycardia
e) Rise in blood pressure

\section{Question 4}

What is the percentage risk of recurrent hemorrhage after a Forrest Ilb bleed without local endoscopic treatment?
(a) $5 \%$ to $10 \%$
(b) $15 \%$ to $20 \%$
(c) $25 \%$ to $30 \%$
(d) $35 \%$ to $40 \%$
(e) $45 \%$ to $50 \%$

\section{Question 5}

Which of the following statements about local endoscopic therapy for gastrointestinal bleeding is correct?

a) Local endoscopic therapy reduces the need for surgery.

b) Local endoscopic therapy is of lesser importance than surgical treatment.

c) Local endoscopic therapy is limited to the application of one particular method.

d) Local endoscopic therapy can only be performed in the esophagus.

e) Local endoscopic therapy is performed through a rigid endoscope.

\section{Question 6}

Which of the following statements about medical therapy for gastrointestinal bleeding is correct?

a) Antibiotic prophylaxis after esophageal variceal bleeding improves survival.

b) Antibiotic prophylaxis after gastric ulcer bleeding is indicated for patients over 80 years of age. c) Treatment with a proton pump inhibitor improves survival in patients with esophageal variceal bleeding.

d) A Forrest lla hemorrhage is treated with intravenous antibiotics.

e) The administration of terlipressin causes arterial vasodilation in patients with gastrointestinal hemorrhage.

\section{Question 7}

Which of the following techniques is used during endoscopic hemostasis?

a) The injection of hyaluronic acid

b) The application of endoclips

c) The application of tamponades

d) The injection of botulinum toxin solution

e) Embolization with starch spheres

\section{Question 8}

Which of the following parameters indicates a poor prognosis ?

a) Advanced age of the patient

b) Uncomplicated ventricular ulcer

c) Helicobacter pylori colonization

d) Stable blood pressure

e) No active bleeding seen at endoscopy

\section{Question 9}

Which of the following statements about varices is correct?

a) Varices of the gastric fundus are surgically removed in a very invasive operation on the stomach.

b) The placement of a balloon catheter is the treatment of choice for variceal hemorrhage.

c) A Mallory-Weiss lesion is a varix of the duodenum.

d) Esophageal varices are often the cause of upper gastrointestinal hemorrhage.

e) For a patient with variceal bleeding, endoscopy should be deferred until the bleeding stops.

\section{Question 10}

Which of the following statements about the eradication of an associated Helicobacter pylori infection in a patient with a gastrointestinal hemorrhage is correct ?

a) The eradication of an associated Helicobacter pylori infection is the treatment of choice for esophageal varices if no other source of hemorrhage is found.

b) The successful eradication of an associated Helicobacter pylori infection reduces the risk of recurrent hemorrhage in the first year after the initial bleed to less than $5 \%$.

c) Helicobacter pylori eradication after a Mallory-Weiss tear raises the probability of having no further hemorrhages by $60 \%$.

d) Randomized studies have refuted the hypothesis that successful Helicobacter pylori eradication after bleeding from ulcers is an effective treatment for this disease.

e) The eradication of an associated Helicobacter pylori infection is not indicated in patients who are taking non-steroidal anti-inflammatory drugs. 